\title{
Self-diffusion on copper surfaces
}

Hansen, L.; Stoltze, Per; Jacobsen, Karsten Wedel; Nørskov, Jens Kehlet

Published in:

Physical Review B

Link to article, DOI:

10.1103/PhysRevB.44.6523

Publication date:

1991

Document Version

Publisher's PDF, also known as Version of record

Link back to DTU Orbit

Citation (APA):

Hansen, L., Stoltze, P., Jacobsen, K. W., \& Nørskov, J. K. (1991). Self-diffusion on copper surfaces. Physical Review B, 44(12), 6523-6526. https://doi.org/10.1103/PhysRevB.44.6523

\section{General rights}

Copyright and moral rights for the publications made accessible in the public portal are retained by the authors and/or other copyright owners and it is a condition of accessing publications that users recognise and abide by the legal requirements associated with these rights.

- Users may download and print one copy of any publication from the public portal for the purpose of private study or research.

- You may not further distribute the material or use it for any profit-making activity or commercial gain

- You may freely distribute the URL identifying the publication in the public portal

If you believe that this document breaches copyright please contact us providing details, and we will remove access to the work immediately and investigate your claim. 


\title{
Self-diffusion on copper surfaces
}

\author{
L. Hansen, P. Stoltze, K. W. Jacobsen, and J. K. Nørskov \\ Laboratory of Applied Physics, Technical University of Denmark, DK-2800 Lyngby, Denmark
}

(Received 25 February 1991)

\begin{abstract}
The diffusion paths and activation energies of a $\mathrm{Cu}$ adatom on $\mathrm{Cu}(100), \mathrm{Cu}(111)$, and $\mathrm{Cu}(110)$ are studied using the effective-medium theory to calculate the energetics. For the (100) and (110) faces, diffusion via an exchange mechanism is found to be important. The transition state for these paths is stabilized by a direct covalent interaction as proposed by Feibelman. On the (111) face and for diffusion along the close-packed rows on the (110) surface, a simple hopping mechanism is found to be most favorable.
\end{abstract}

Recently it has been suggested that the self-diffusion of metal atoms adsorbed on the (100) surface of the metal itself diffuses by a more complex route than would naively be assumed. ${ }^{1-3}$ Adatoms on a fcc (100) surface usually have equilibrium position in the fourfold hollow sites. Field emission experiments on $\operatorname{Ir}(100)$ and $\operatorname{Pt}(100)$ strongly suggest that the diffusion between two adjacent hollow sites on the surface occurs by exchange with one of the atoms in the surface, rather than by the simpler diffusion path over the twofold-coordinated bridge site. ${ }^{2,3}$ The experimental investigations were prompted by the results of a local-density calculation for $\mathrm{Al}$ adatoms on $\mathrm{Al}(100),{ }^{1}$ showing that the exchange process has a lower activation barrier than the simple bridge hopping process. Moreover, the calculation suggested that the origin of this effect is a strong covalent interaction between the diffusing adatom and the surface atoms along the diffusion path.

In this Brief Report we investigate this effect further. Using the effective-medium theory to calculate the energetics of a diffusing $\mathrm{Cu}$ adatom on a $\mathrm{Cu}(100)$ surface, we show that the same effect is present on copper. We show the effect to be directly linked to strong bondingantibonding splittings in the one-electron spectrum and thus support Feibelman's picture ${ }^{1}$ that it is a covalent bonding effect. Taking advantage of the simplicity of the total-energy method employed, we are able to map out the energetics of the full diffusion path, and to make a systematic investigation of the role of similar processes on the other low index $\mathrm{Cu}$ surfaces. We find that on $\mathrm{Cu}(111)$ simple adatom hopping is always facile and the exchange process is very unfavorable. On $\mathrm{Cu}(110)$ simple hopping along the troughs between the close-packed rows is the energetically most favorable process, but for diffusion across the rows an exchange process is again energetically preferred. This is in qualitative agreement with experiment on fcc (110) surfaces. ${ }^{4}$

The systems studied consist of a semi-infinite surface with a $10 \times 10$ surface unit cell holding one adatom. To study diffusion in, say, the $x$ direction the $x$ coordinate of the adatom is increased in small increments. After each move the $y$ and $z$ coordinates of the diffusing atom and all the coordinates of the nearest-neighbor surrounding atoms (typically 15 atoms) are varied to minimize the total energy. When studying exchange processes it is often better to force the center of mass of the adatom and the surface atom that it exchanges place with rather than the adatom itself. Care has been taken that the energy minimization routine has not made discontinous, unphysical jumps in some of the coordinates.

The total energy for each configuration is calculated using the effective-medium theory. ${ }^{5}$ This gives an approximate expression for the total energy which is very convenient in cases like the present where large numbers of atoms and many different atomic configurations are treated. The expression for the total energy which can be derived from density-functional theory is ${ }^{5}$

$$
E_{\mathrm{total}}=\sum_{i} E_{c}\left(\bar{n}_{i}\right)+\sum_{i} \Delta E_{\mathrm{AS}}^{(i)}+\Delta E_{1-\mathrm{el}}
$$

where the sum is over all the atoms in the system. The influence of the metallic surroundings on an atom is expressed as a function of one quantity, the embedding density $\bar{n}$, which is the average electron density contributed by surrounding atoms in a neutral sphere around the atom. The function $E_{c}(\bar{n})$ is calculated once and for all from first principles by embedding one atom in a homogeneous electron gas. The atomic-sphere correction $\Delta E_{\mathrm{AS}}$ describes the difference in the electrostatic interaction between the atoms in the system under consideration and in a close-packed (fcc) arrangement [where the electrostatic interactions are already included in the $E_{c}(\bar{n})$ function].

The first two terms of (1) and the parameters used are described in detail elsewhere. ${ }^{5}$ These two terms are sufficient to give a good description of bulk $\mathrm{Cu}$ and of many properties of $\mathrm{Cu}$ surfaces including adsorbate-induced reconstructions. ${ }^{6}$ The main aspect of the present application is that we include the last term in (1), which is usually small, but turns out to be very important for proper description of the exchange diffusion process. The last term in (1) involves the difference in the one-electron spectrum in the system under study and in a fcc crystal of the metal with the same $\bar{n}$ :

$\Delta E_{1-\mathrm{el}}=\sum_{i} \int^{\varepsilon_{F}} n_{i}(\varepsilon) \varepsilon d \varepsilon-\sum_{i} \int^{\varepsilon_{F}} n_{i}^{\mathrm{fcc}}(\varepsilon) \varepsilon d \varepsilon$. 
Here $n_{i}(\varepsilon)$ and $n_{i}^{\text {fcc }}(\varepsilon)$ are the projected density of states on atom $i$ in the real system and in a fcc crystal of the same $\bar{n}$, respectively. We use a simple tight-binding Hamiltonian $H$ to evaluate $\Delta E_{1 \text {-el }}$. The matrix elements $H_{i j}$ between atomic orbitals on atoms $i$ and $j$ are taken to depend only on the interatomic distance $r_{i j}$. Since the matrix elements will be roughly proportional to the amplitude of orbital $i$ at site $j$ and vice versa, $H_{i j}$ is taken to have a radial dependence proportional to $\left(\bar{n}_{i}^{(j)}\right)^{1 / 2}$ where $\bar{n}_{i}^{(j)}$ is the contribution to $\bar{n}_{i}$ from atom $j$ :

$$
H_{i j}=-b \exp \left(-\frac{\eta_{2}}{2}\left(r_{i j}-\beta s_{0}\right)\right) \text {. }
$$

Here $r_{i j}$ is the distance between atoms $i$ and $j, \eta_{2}$ is the falloff constant of the electron density, and $\beta s_{0}$ is the equilibrium lattice constant of $\mathrm{Cu}\left(s_{0}\right.$ is the equilibrium Wigner-Seitz radius). The prefactor $b=0.60 \mathrm{eV}$ is a real and positive constant as we are dealing with $s$ orbitals only, and it is determined by equating the bandwidth of the fcc equilibrium structure to the result published by Moruzzi, Janak, and Williams ${ }^{7}$ for the $\mathrm{Cu} 4 s$ band in their self-consistent band-structure calculation. ${ }^{8}$

A simple $s$-band tight-binding model is sufficient for the present purposes for two reasons. First of all, only the $s$ band is partly filled. The $\mathrm{Cu} d$ bands are completely filled and the $p$ states are well above the Fermi level and completely empty. Second, the simple model is only used to calculate the difference between the oneelectron energy in the real system and in the reference system. This means that even though the model is crude the absolute error introduced in the calculated energy is small. The $s$ - $d$ coupling, for instance, is thus included in the total energy by the fact that it is included in the energy of the reference system [the $E_{c}$ term in (1)] and only the difference in $s$ - $d$ coupling between the reference system and the real system is neglected.

In evaluating $\Delta E_{1 \text {-el }}$ we make use of the work by Brown and Carlsson. ${ }^{9}$ By calculating the sum of the one-electron energies $E_{1}$ in a simple one-band nearest-neighbor tightbinding model for a large number of different structures, they show that $E_{1}$ can be expressed in terms of one quantity, $\gamma_{4}=\mu_{4} / \mu_{2}^{2}$ which is the ratio of the fourth moment of the local density of states at an atom and the square of the second moment:

$$
E_{1}=\left[E_{1}^{0}+A\left(\gamma_{4}-\gamma_{4}^{0}\right)\right] \sqrt{\mu_{2}} .
$$

(4) can be viewed as the lowest-order expansion of $E_{1}$ in powers of $\gamma_{4}$ around a reference structure with oneelectron energy $E_{1}^{0}$ and $\gamma_{4}=\gamma_{4}^{0}$, and what Brown and Carlsson have shown is that the first-order term dominates over a very large range of $\gamma_{4}$ 's. In this expansion there will also be terms proportional to the other $\gamma_{n}$ 's given by the $n$th moments of the local density of states, but these terms are much smaller than the fourth moment contribution. The $\gamma_{3}$ contribution is thus considerably smaller than the one from $\gamma_{4}$. The coefficient $A$ depends on the degree of filling of the band. $A$ is largest for a half-filled band, $f=0.5$ and decreases monotonically for smaller and larger $f$. The physical content of (4) is that, for a given second moment (or bandwidth) $\mu_{2}$, the bonding is strongest ( $E_{1}$ smallest) if the density of states is double peaked, with a clear splitting between the bonding and antibonding states and, at the same time the band is half filled so that only the bonding states are filled. The smallest value $\gamma_{4}$ can take is one, which is found for the dimer (which has sharp bonding and antibonding states) whereas the close-packed fcc structure has the largest value of 3.75 . Between these two extremes one finds, for instance, the bcc and diamond structure.

Combining (4) with (2) we get

$$
\Delta E_{1-\mathrm{el}}=A \sum_{i}\left(\gamma_{4, i}-\gamma_{4, \mathrm{fcc}}\right) \sqrt{\mu_{2, i}},
$$

where the sum is over the atoms in the system. For $\mathrm{Cu}$ which has a half-filled $s$ valence band, $A=0.1 .^{9}$ In the calculation of the matrix elements $H_{i j}$ we have used a Fermi function cutoff between first and second neighbors in the fcc structure, since the method above is developed explicitly for a nearest-neighbor model. This approximate, but computationally efficient, method is sufficient for the present exploratory study, but can of course in principle be improved if necessary.

The results of the calculations are summarized in Fig. 1. Consider first the (100) surface. The energy along the bridge and the exchange diffusion path are shown and the calculated exchange diffusion path is also illustrated in the figure. The exchange path is seen to have a substantially lower activation barrier than the other path. This is in good qualitative agreement with the experimental observations ${ }^{2,3}$ and with the calculations of Feibelman. ${ }^{1}$ The present results also resemble those of Feibelman with respect to the geometry of the transition state. We find that in the transition state (cf. Fig. 1) the distance between the two $\mathrm{Cu}$ atoms that change place is $9.7 \%$ smaller than in bulk $\mathrm{Cu}$ and the distance beween these atoms and the nearest neighbors in the surface plane is reduced by $10.8 \%$. In the full local-density calculation of Feibelman ${ }^{1}$ the same figures are $7.8 \%$ and $11.3 \%$, respectively. Feibelman only tried one transition state geometry. The present calculation of the whole diffusion path confirms his choice.

For the $\mathrm{Cu}(100)$ surface we also include in Fig. 1 the energy variation along the two diffusion paths when $\Delta E_{1-\mathrm{el}}=0$. It is clearly seen that the exchange path does not have the lowest energy any more, showing that it is the one-electron energy difference that is responsible for the effect.

The one-electron correction only contributes significantly if $\gamma_{4}$ is much smaller than $\gamma_{4, \mathrm{fcc}}$. For the usual configurations encountered in the metal and at the surface this is not the case. $\Delta E_{1-\text { el }}$ thus contributes only 0.05 $\mathrm{eV}$ per surface atom to the (100) surface energy. But for configurations where, for instance, two atoms come very close to each other, one of the hopping matrix elements starts to dominate and $\gamma_{4}$ approaches the value for the dimer. Under most circumstances $\Delta E_{1 \text {-el }}$ can thus be neglected, but for exactly the kind of situations encountered during the exchange process, where the two moving atoms get very close to each other and to the atoms underneath (cf. Fig. 1), $\Delta E_{1-\mathrm{el}}$ becomes significant. The underlying reason is that for these configurations distinct bonding and antibonding states are developed and only 
the bonding ones are filled. Feibelman ${ }^{1}$ arrived at the same conclusion by looking at the resulting charge densities.

In Fig. 1 we also include results for adatom diffusion on the $\mathrm{Cu}(111)$ and $\mathrm{Cu}(110)$ surfaces. On the close-packed (111) surface the barrier for diffusion is very low for the ordinary hopping process, because during this process

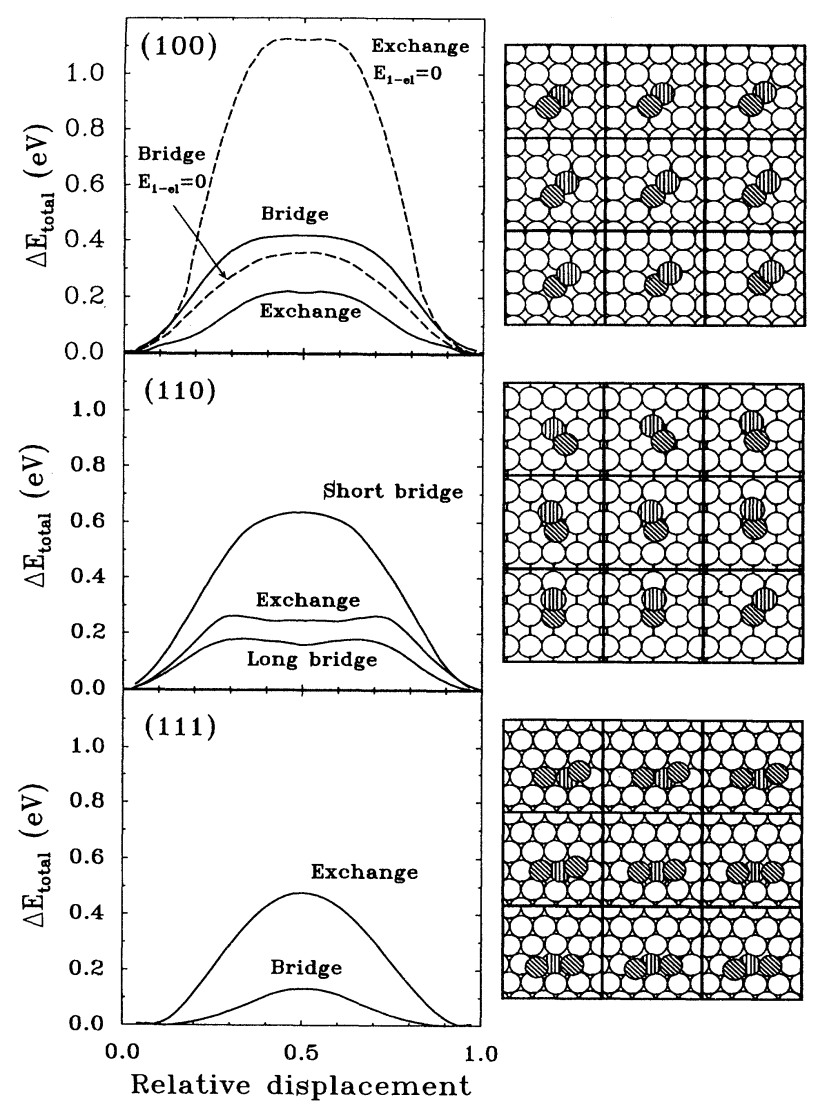

FIG. 1. Calculated energy change along the diffusion path of a $\mathrm{Cu}$ adatom on $\mathrm{Cu}(100), \mathrm{Cu}(111)$, and $\mathrm{Cu}(110)$. The starting point for the diffusion process is the adatom in the equilibrium position, which in all cases in the highest coordination number site on the surface [fourfold-, threefold-, and fivefold-coordinated for the (100), (111), and (110) faces, respectively]. All nearest-neighbor coordinates are allowed to relax along the path. In all three cases results are shown for the simple bridge hopping and for the exchange process. The calculated path for the exchange process is shown to the right of the corresponding energy curves. For the exchange process on the (110) surface no preference was found between the path shown and the one where the adatom ends up in the site diagonal to the starting point. For the (100) surface the results of a similar calculation with $\Delta E_{1-\mathrm{el}}=0$ is also included to show the importance of the one-electron correction for the exchange process. For the (110) surface, there are three diffusion paths considered, hopping along (over the long-bridge sites) and across (over the short-bridge sites) the close-packed rows, and an exchange process for diffusion across the rows. All attempted exchange processes along row diffusion gave much higher activation energies. the adatom only changes its coordination number by one, from three at the equilibrium site on the surface to two at the bridge site. The configuration at the bridge site is not very different from that on the (100) surface, so the much smaller barrier for overbridge hopping on the (111) surface is not due to a particularly stable transition state, but rather due to a starting point which is more unstable than on the (100) face. The energy of a $\mathrm{Cu}$ adatom on the (111) face is $0.75 \mathrm{eV}$ relative to the energy in the bulk, whereas for the (100) surface the similar energy is $0.43 \mathrm{eV}$. The atoms in the surface layer are so stable on this surface that the exchange process has much too large an energy.

The very facile diffusion on fcc (111) surfaces has been observed both with field ion microscopy ${ }^{10}$ and using electron microscopy. ${ }^{11}$ The activation energies found in these studies are in the range $0.1-0.15 \mathrm{eV}$ as found in the present study.

On the (110) surface the situation is a little more complex due to the lower symmetry of this face. There are two inequivalent diffusion paths, along the close-packed rows in the $\langle 1 \overline{1} 0\rangle$ direction (over the long-bridge sites), and across the close-packed rows in the $\langle 001\rangle$ direction (over the short-bridge sites). Hopping along the closepacked rows is seen in Fig. 1 to have a low barrier, again because the coordination number does not change much along the diffusion path. For diffusion over the closepacked rows the barrier is large. Here the transition state is a twofold-coordinated bridge site like on the other surfaces and the large stability of an adatom in the fivefoldcoordinated equilibrium site on the (110) surface (only $0.36 \mathrm{eV}$ less stable than in the bulk) makes the barrier large. This again makes the exchange process a likely candidate, because the system can increase the bonding contribution from the $\Delta E_{1-\text { el }}$ term in the transition state in the same way as on the (100) surface. An important difference from the (100) surface is that while $\Delta E_{1-e l}$ helps stabilize the transition state for the exchange process, the exchange process is energetically favorable even without $\Delta E_{1-\text { el }}$ included.

Preferential diffusion along the close-packed rows has been observed on some fcc (110) surfaces ${ }^{10}$ and the role of the exchange mechanism for cross channel diffusion has been illustrated by the diffusion of $W$ adatoms on $\operatorname{Ir}(110)$ where it was found that when an adatom was observed in an adjacent row after a diffusion event, it was an Ir atom from the substrate and not the $\mathrm{W}$ atom. ${ }^{4}$

It must be noted that the present calculations are only for low-temperature diffusion. At temperatures approaching the melting point, where the self-diffusion on surfaces has also been studied, ${ }^{12}$ the picture is much more complex because the substrate on which the adatom diffuses is no longer ordered. ${ }^{13}$

In conclusion, we have shown that the exchange process is important for adatom self-diffusion on $\mathrm{Cu}(100)$ and $\mathrm{Cu}(110)$ surfaces. In accordance with the findings of Feibelman, ${ }^{1}$ the effect is closely connected with a strong covalent bonding in the transition state and in the effective-medium theory and related methods one can describe the effect properly by including the one-electron correction which is usually negligible for metal surfaces. 
For diffusion on $\mathrm{Cu}(111)$ and along the close-packed rows of $\mathrm{Cu}(110)$ the barrier for the simple hopping process is so low and the surface atoms so stable that the exchange process is not found to be energetically favored.

Note added in proof. After submission of the manuscript, the authors became aware of an experimental determination of the activation energy for adatom self-diffusion on $\mathrm{Cu}(100)$ by H.-J. Ernst, F. Fabre, and J. Lapujoulade (unpublished). They determine an activation energy of $0.28 \pm 0.06 \mathrm{eV}$, in excellent agreement with the one determined by the calculation. In addition, Lapujoulade has brought to our attention a determination of the ratio of the diffusivities parallel and perpendicular to the close-packed rows on $\mathrm{Cu}(110)$ by J. Cousty,
R. Peix, and B. Perraillon [Surf. Sci. 107, 586 (1981)]. They determine this ratio to be $4.3 \pm 1$ at $810 \mathrm{~K}$. Using the difference in activation energy from Fig. 1 of $0.09 \mathrm{eV}$ and assuming the same preexponential factor for the two processes, the calculation gives a ratio of 3.5 at this temperature. Again both the sign and absolute magnitude are in good agreement with experiment. We are grateful to Dr. Ernst and Dr. Lapujoulade for communicating these results to us.

The authors have benefited from the help of Ole Bøssing Christensen and from financial support from the Danish Research Councils through the Center for Surface Reactivity.
${ }^{1}$ P. J. Feibelman, Phys. Rev. Lett. 65, 729 (1990).

${ }^{2}$ G. Kellogg and P. J. Feibelman, Phys. Rev. Lett. 64, 3143 (1990).

${ }^{3}$ C. Chen and T. T. Tsong, Phys. Rev. Lett. 64, 3147 (1990).

${ }^{4}$ J. D. Wrigley and G. Ehrlich, Phys. Rev. Lett. 44, 661 (1980).

${ }^{5}$ K. W. Jacobsen, J. K. Nørskov, and M. J. Puska, Phys. Rev. B 35, 7423 (1987); K. W. Jacobsen, Comments Condens. Matter Phys. 14, 129 (1988).

${ }^{6} \mathrm{~K}$. W. Jacobsen and J. K. Nørskov, Phys. Rev. Lett. 65, 1788 (1990).

${ }^{7}$ V. L. Moruzzi, J. F. Janak, and A. R. Williams, Calculated Electronic Properties of Metals (Pergamon, New York, 1978).
${ }^{8}$ The same procedure has been employed in a study of small $\mathrm{Cu}$ clusters, where $\Delta E_{1 \text {-el }}$ is also important. See, O. B. Christensen, K. W. Jacobsen, J. K. Nørskov, and M. Manninen, Phys. Rev. Lett. 66, 2219 (1991).

${ }^{9}$ R. H. Brown and A. Carlsson, Phys. Rev. B 32, 6125 (1985).

${ }^{10}$ For a review, see G. Ehrlich, in MRS Symposia Proceedings No. 57 (Materials Research Society, Pittsburgh, 1987), p. 313.

${ }^{11}$ G. W. Jones, J. Macano, J. K. Nørskov, and J. A. Venables, Phys. Rev. Lett. 65, 3317 (1990).

${ }^{12}$ J. W. M. Frenken, J. P. Toennies, and Ch. Wöll, Phys. Rev. Lett. 60, 1727 (1988).

${ }^{13}$ P. Stoltze and J. K. Nørskov (unpublished). 